February 8, $2020 \quad$ 0:59 WSPC/INSTRUCTION FILE semimag16.jk

International Journal of Modern Physics B

(C) World Scientific Publishing Company

\title{
Tunneling between bilayer quantum Hall structures in a strong magnetic field
}

\author{
F. D. Klironomos \\ Department of Physics, University of Florida, P.O. Box 118440 \\ Gainesville, Florida 32611-8440, USA \\ fkliron@phys.ufl.edu \\ A. T. Dorsey \\ Department of Physics, University of Florida, P.O. Box 118440 \\ Gainesville, Florida 32611-8440, USA \\ dorsey@phys.ufl.edu
}

Received 23/06/2004

Revised 16/08/2004

\begin{abstract}
We calculate the tunneling current in a quantum Hall bilayer system in the strong magnetic field limit. We model the bilayer electron system as two Wigner crystals coupled through interlayer Coulomb interactions, treated in the continuum limit. We generalized the Johansson and Kinaret (JK) model $^{1}$ and were able to study the effect of the low energy out-of-phase magnetophonon modes produced as a result of tunneling events. We find the same scaling behavior of the tunneling current peak with the magnetic field as found by JK but were able to find the tunneling current scaling behavior with interlayer distance as well.
\end{abstract}

Keywords: quantum Hall bilayer;two dimensional electron system

\section{Introduction}

A two-dimensional bilayer electron system under the presence of a strong perpendicular magnetic field exhibits a Coulomb barrier peak in the tunneling current in transport experiments ${ }^{2}$. This peak is pronounced in high density samples and is attributed to the highly correlated state of the electron liquid where the cyclotron motion frustrates the ability of local electrons to respond to a tunneling event. A necessary amount of energy of the order of $e^{2} / 4 \pi \epsilon\langle a\rangle$, where $\langle a\rangle$ is the average interelectron distance, has to be provided to the system to collectively respond to tunneling.

Our model describes this tunneling behavior and is based on the Johansson and Kinaret (JK) model ${ }^{1}$ which assumes that the two layers of electrons are in commensurate Wigner crystal states. A tunneling event disrupts the Wigner crystals producing collective fluctuations (magnetophonons) that dissipate the energy of the tunneling electron and smooth-out the local charge-defect associated with it. We 
work in the continuum limit treating the whole system as an elastic medium. We should stress out that the actual state of the system is not a Wigner crystal but a rather highly correlated liquid state. Our work differs from the JK model in the fact that we have added interlayer Coulomb interaction, correlating the two layers, and additionally we were able to produce analytic results as well as numerical ones. Our results are summarized in Fig. (1).

\section{Bilayer theory}

We assume that each layer of electrons is a two-dimensional elastic medium interacting via Coulomb interaction (intralayer). Each electron has a displacement associated with its equilibrium position in the Wigner crystal which becomes the displacement field in the continuum limit. The magnetic field couples the transverse and longitudinal modes into gapless magnetophonon and gapped (by the cyclotron frequency) magnetoplasmon modes. These correspond to the internal modes of each layer when they are far apart (JK model). When the layers are brought within proximity the interlayer Coulomb interaction will introduce an additional coupling of those four modes producing two in-phase and two out-of-phase modes. The in-phase modes resemble single layer modes but the out-of-phase modes are gapped by the energy cost to shear the commensurate Wigner crystals. This energy cost is associated with the penalty the short range part of the interlayer Coulomb interaction introduces to an out-of-phase motion of the two Wigner crystals. Additionally, a tunneling electron couples only to out-of-phase modes. This happens because during tunneling the positive charge defect the electron leaves behind has to be "coveredup" while the receiving layer has to create a place for the incoming electron by "opening-up" resulting in a relative out-of-phase motion of the two crystals. The total Hamiltonian for the bilayer system assumes the form,

$$
\begin{aligned}
H & =H_{0}+H_{T}^{-}+H_{T}^{+} \\
& =\left[\epsilon_{A}+i \sum_{s} M_{s A}\left(a_{s}^{\dagger}-a_{s}\right)\right] c_{A}^{\dagger} c_{A}+\left[\epsilon_{B}+i \sum_{s} M_{s B}\left(a_{s}^{\dagger}-a_{s}\right)\right] c_{B}^{\dagger} c_{B} \\
& +\sum_{s} \hbar \Omega_{s} a_{s}^{\dagger} a_{s}+T c_{A}^{\dagger} c_{B}+T c_{B}^{\dagger} c_{A},
\end{aligned}
$$

where $\epsilon_{A}, \epsilon_{B}$ are the corresponding Madelung energies for the two Wigner crystals, $a_{s}^{\dagger}, a_{s}$ are the creation and annihilation operators for the collective electron modes while $c^{\dagger}, c$ are the tunneling electron's creation and annihilation operators for the two layers. The ground state of this Hamiltonian is a bath of collective modes coupled to an external electron present in the system. Tunneling is an independent single particle process where the tunneling matrix element $T$ is assumed to be real and calculated as in $\mathrm{JK}^{1}$. The tunneling current for different bias voltage $V$ is given by

$$
I(V)=\frac{e}{\hbar^{2}} \int_{-\infty}^{+\infty} d t e^{i e V t / \hbar}\left\langle\left[H_{T}^{-}(t), H_{T}^{+}(0)\right]\right\rangle
$$


We calculate the zero-temperature limit for the correlation function, since the experimental temperature range is $0.1 \mathrm{~K} \sim 10^{-5} \mathrm{eV}$ while the applied bias voltage is in the $\mathrm{mV}$ range. Additionally we concentrate on the magnetophonon modes only that are responsible for the low bias Coulomb barrier peak. We find that the correlation function $\left\langle H_{T}^{-}(t) H_{T}^{+}(0)\right\rangle \sim C(t)$ can be Fourier transformed and obeys an integral equation of the form

$$
\omega C(\omega)=\int_{0}^{1} d x f(x) \omega(x) C(\omega-\omega(x)),
$$

where $\omega(x)$ is the magnetophonon dispersion relation and $f(x)$ is a weight function. Equation (3) has an approximate large frequency solution of the form

$$
C(\omega) \sim \exp \left\{-\frac{\left(\omega-c_{1}\right)^{2}}{2 c_{2}}\right\}
$$

where $\sqrt{c_{2}} \sim 1 / a_{0}^{2} \sqrt{B}$ for layers far apart and $c_{2} \sim d / a_{0}^{3} \sqrt{B}$ for layers close together, where $d$ is the interlayer distance and $a_{0}$ the Wigner crystal lattice parameter. Based on that we propose a trial solution of the form

$$
C(\omega)=N \omega^{r} e^{-\lambda \omega^{2}},
$$

where $N, r, \lambda$ are self-consistently evaluated from the properties of $C(t)$ namely,

$$
\begin{array}{r}
\int_{0}^{\infty} d \omega C(\omega)=2 \pi \\
\int_{0}^{\infty} d \omega \omega C(\omega)=2 \pi c_{1} \\
\int_{0}^{\infty} d \omega \omega^{2} C(\omega)=2 \pi\left(c_{2}+c_{1}^{2}\right) .
\end{array}
$$

Our solutions are presented in Fig. (1) for different applied magnetic field values. For all our model parameters we have used the experimental system reported in Ref. 2. For the magnetophonon gap value we extracted it from time-dependent Hartree Fock calculations of the magnetophonon dispersion relation for the bilayer system $^{4}$. We numerically integrated Eq. (3) to verify the validity of our analytic solution with very satisfactory results.

\section{Conclusions}

We calculated the tunneling current curve for a bilayer quantum Hall system under the presence of a strong perpendicular magnetic field and reproduced the Coulomb barrier peak experimentally found. Our model assumed that the two dimensional electron systems were in Wigner crystal states and calculated the eigenmodes in the continuum approximation where the two layers are treated as elastic media. We found that by introducing an interlayer Coulomb interaction the internal modes of the system couple to in-phase and out-of-phase ones. The latter acquire a gap in the magnetophonon branch associated with the short-range interlayer correlations built 


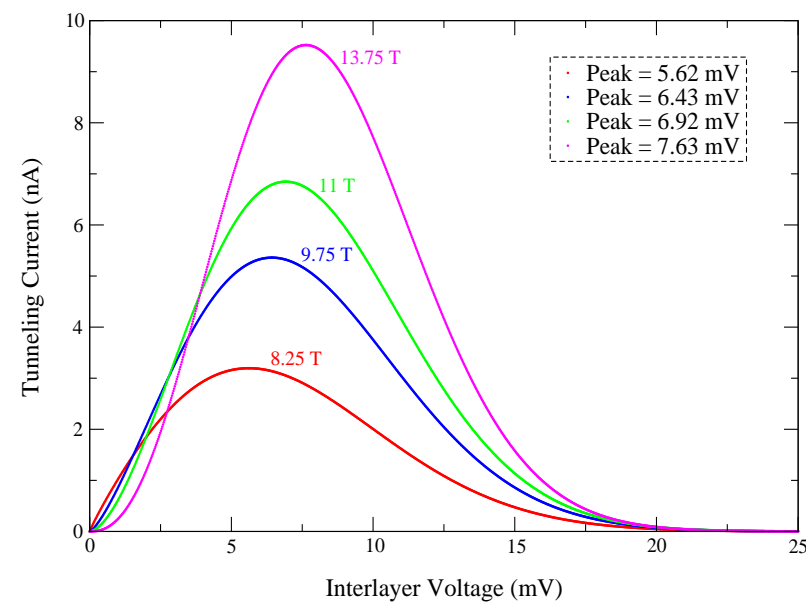

Fig. 1. Analytic results for the tunneling current of a two-dimensional bilayer system for different magnetic field values. The legend shows the corresponding peak bias voltage.

into the system by the Coulomb force. This magnetophonon branch provides the necessary collective mode coupling for tunneling electrons to dissipate their energy, and for the system to relax the charge defect a tunneling event creates. We found the same scaling behavior JK report for the tunneling curve but additionally were able to extract the dependence on interlayer distance as well.

\section{Acknowledgments}

We would like to thank S. M. Girvin for valuable discussion on the bilayers and René Côté and collaborators for providing the magnetophonon dispersion curves. This work was supported by NSF DMR-9978547.

\section{References}

1. P. Johansson and J. M. Kinaret, Phys. Rev. B50, 4671 (1994).

2. J. P. Eisenstein, L. N. Pfeiffer, and K. W. West Phys. Rev. Lett. 69, 3804 (1992).

3. L. D. Landau and E. M. Lifshitz, in Theory of Elasticity (Butterworth-Heinemann, 3rd Edition), p. 9.

4. René Côté et al., private communication. 